\begin{tabular}{c|c|c|}
\cline { 2 - 3 } & REVISTA SABERES APUDEP & Volumen 3 Número 1 \\
\hline
\end{tabular}

\title{
ÁREAS VERDES DEL CENTRO REGIONAL UNIVERSITARIO DE COLÓN, UNIVERSIDAD DE PANAMÁ, ESPACIOS NATURALES EN RIESGO
}

\author{
GREEN AREAS OF THE CENTRO REGIONAL UNIVERSITARIO DE COLÓN, \\ UNIVERSIDAD DE PANAMÁ, NATURAL SPACES AT RISK.
}

Félix Antonio Biens Bethancourt y Vera De la Cruz Cabrera

Universidad de Panamá, Centro Regional Universitario de Colón, Departamento de Ciencias Ambientales, Biología Ambiental, e-mail: felix 15@yahoo.es vera.delacruz@up.ac.pa

\section{RESUMEN}

Las áreas verdes en los centros de estudios universitarios desempeñan un papel fundamental, ya que son espacios que, debido a sus características naturales intrínsecas, ofrecen servicios ambientales, sirven como espacios abiertos que conducen al aprendizaje y funcionan como puntos de experimentación convirtiéndose en verdaderos laboratorios para diferentes disciplinas. Gestionar un área verde implica no solo la conservación del contacto con la naturaleza, sino también sensibilizar a la comunidad universitaria sobre la importancia de su atención y manejo, lo que requiere un plan de gestión ambiental que involucre a los diferentes actores para aprovechar estas áreas en beneficio de todos. Esto requiere, además, de una revisión que brinde información actualizada para un diseño sostenible, que permita determinar un uso óptimo, evidenciando que la adecuación es necesaria. Este estudio se basó en la revisión del inventario florístico de árboles entre 2012 y 2013 en las 14.9 hectáreas del Centro Regional Universitario de Colón de la Universidad de Panamá, ubicando en el campo las especies, además de la observación de crecidas sin control de herbazales, el deterioro de la infraestructura debido al crecimiento de la vegetación en áreas inapropiadas, la distribución irregular de árboles mediante georreferenciación, creando un mapa con los datos actualizados recopilados, evaluando su estado de salud (hongos, insectos, infecciones y / o daños mecánicos) con el fin de obtener información de primera mano para proponer una gestión adecuada según el caso (tala, reemplazo, reubicación o poda), al proporcionar un informe actualizado que sirva de base, teniendo en cuenta las áreas y los árboles que están en riesgo y que deben protegerse $o$ aquellos que puedan ser un peligro para la comunidad universitaria.

PALABRAS CLAVE: Áreas verdes, educación ambiental, gestión ambiental, conservación, reforestación 


\begin{tabular}{|c|c|c|}
\cline { 2 - 3 } & REVISTA SABERES APUDEP & Volumen 3 Número 1 \\
\hline
\end{tabular}

\begin{abstract}
The green areas in the centers of university studies play a fundamental role, since so spaces that, due to their intrinsic natural characteristics, offer environmental services, serve as open spaces that lead to learning and function as experimentation points becoming true laboratories for Different disciplines Managing a green area implies not only the conservation of contact with nature, but also sensitizing the university community about the importance of their attention and management, which requires an environmental management plan that involves the different actors to take advantage of these areas in benefit of all. This also requires a review that provides upto-date information for a sustainable design, which makes it possible to determine optimal use, evidencing that adequacy is necessary. This study was based on the review of the floristic inventory of trees between 2012 and 2013 in the 14.9 hectares of the Centro Regional Universitario de Colón of the Universidad de Panamá, placing the species in the field, in addition to the observation of floods without control of grasslands, the deterioration of the infrastructure due to the growth of vegetation in inappropriate areas, the irregular distribution of trees by georeferencing, creating a map with the updated data collected, assessing their health status (fungi, insects, infections and / or mechanical damage) with in order to obtain first-hand information to propose appropriate management as appropriate (logging, replacement, relocation or pruning), by providing an updated report that serves as a basis, taking into account the areas and trees that are at risk and that should protect yourself or those that may be a danger to the university community.
\end{abstract}

KEYWORDS: Green areas, environmental education, environmental management, conservation, reforestation

\title{
INTRODUCCIÓN
}

El aumento de la urbanización ha alcanzado terrenos con vegetación, por lo tanto, el entorno natural se ha visto significativamente disminuido siendo de suma importancia poder recuperarlo (Undurraga, 1984; Rodríguez Salgado, 2012). La comunidad es el espacio en el cual se desarrolla la vida cotidiana de la mayoría de las personas y familias; esto lleva a la necesidad de crear un ambiente agradable que proporcione bienestar, salud, y seguridad a todos los habitantes y garantice la armonía para con la naturaleza (Escudero y Gross, 1996; Rodríguez Salgado, 2012). 


\begin{tabular}{|c|c|c|}
\cline { 2 - 3 } & REVISTA SABERES APUDEP & Volumen 3 Número 1 \\
\hline
\end{tabular}

Las áreas verdes ayudan a mejorar la calidad del aire asimilando un importante porcentaje de $\mathrm{CO}_{2}$, generando oxígeno, regulando la temperatura ambiental y contribuyendo a evitar la erosión de los suelos; además aportan otros beneficios como: sitios de esparcimiento, crean espacio de sombra, de recreación y de relajación del estrés que se vive en las ciudades (Caballero Deloya, 1996; De Sedas et al., 2010; Correa et al., 2010), también son un elemento ornamental que proporciona goce visual y por ende una mejor calidad de vida.

Los beneficios ambientales producidos por los árboles son múltiples, entregan beneficios intangibles a los habitantes, animan los sentidos y hacen acogedores los espacios que cubren. Son protagonistas de la biodiversidad de las ciudades ya que constituyen el principal hábitat de las plantas y animales urbanos proporcionando una identidad propia al lugar donde se sitúan (XI Congreso Mundial Forestal, 1997).

La ciudad de Colón cuenta con una gran diversidad de árboles que crecen en condiciones muy adversas para su óptimo desarrollo y otros que causan daños a infraestructuras como edificios, banquetas, conductos eléctricos, de agua potable, telefónicas y drenaje (Farnum et al, 2014; Grey y Deneke, 1992).

El Centro Regional Universitario de Colón (CRUC) de la Universidad de Panamá posee una gran diversidad de árboles y arbustos entre nativos, exóticos e introducidos (Farnum et al, 2014). Las áreas verdes de este Centro juegan un papel importante en la dinámica de aprendizaje en general, ya que funcionan como verdaderos laboratorios tanto para las ciencias naturales como para las sociales, como elementos de aporte a la actividad académica de toda la comunidad universitaria. 


\begin{tabular}{|c|c|c|}
\cline { 2 - 3 } & REVISTA SABERES APUDEP & Volumen 3 Número 1 \\
\hline
\end{tabular}

La adecuada valoración de las funciones que cumplen las áreas verdes con relación a los procesos ecológicos y a los servicios que prestan para el mejoramiento de la calidad de vida, constituye un punto de partida para los planes de una gestión ambiental, ocupando el mismo nivel de importancia, la evaluación de la situación actual de dichas áreas y la percepción que sobre ellas tiene la población. La formulación y puesta en práctica de las estrategias adecuadas para la formación y mantenimiento de áreas verdes urbanas, optimiza la gestión ambiental de las mismas, siendo lo último, el objetivo que deben perseguir las asociaciones e instituciones involucradas y mediante el cual brindan un considerable aporte en el proceso de desarrollo sostenible de nuestro país (Ríos Cáceres et al, 1998).

Este trabajo, de carácter descriptivo, tiene como objetivo revisar las condiciones actuales de las áreas verdes del Centro Regional Universitario de Colón en cuanto a su composición, para tener una base para generar una propuesta de mejora y conservación.

\section{MATERIALES Y MÉTODOS}

Corresponde a una investigación descriptiva, desarrollada entre los meses de agosto a diciembre del año 2019. El área de estudio se ubica en el Centro Regional Universitario de Colón (CRUC) de la Universidad de Panamá (Figura 1), en la provincia de Colón, corregimiento de Cristóbal en la localidad de Arco Iris, área revertida, antigua Escuela Secundaria de Rainbow City. Coordenadas: 9 ${ }^{\circ} 20^{\prime} 00^{\prime \prime} \mathrm{N}$ y $79^{\circ} 54^{\prime} 00^{\prime \prime} \mathrm{O}$. Dicha área comprendió las 14.9 hectáreas, sin embargo, actualmente la zona en la que se encontraba la antigua piscina de la antigua escuela está cerrada por trabajos de construcción. 


\begin{tabular}{|c|c|c|}
\cline { 2 - 3 } & REVISTA SABERES APUDEP & Volumen 3 Número 1 \\
\hline
\end{tabular}

Dicho estudio se basó en la revisión del inventario florístico realizado entre los años 2012 y 2013. Además, de la revisión por observación de la distribución irregular arbórea, la crecida sin control de herbazales, el deterioro de infraestructuras por crecimiento de vegetación en zonas inapropiadas y la distribución irregular de árboles mediante georreferenciación. Cómo resultados de esta revisión se diseñó un mapa con los datos recopilados actualizados.

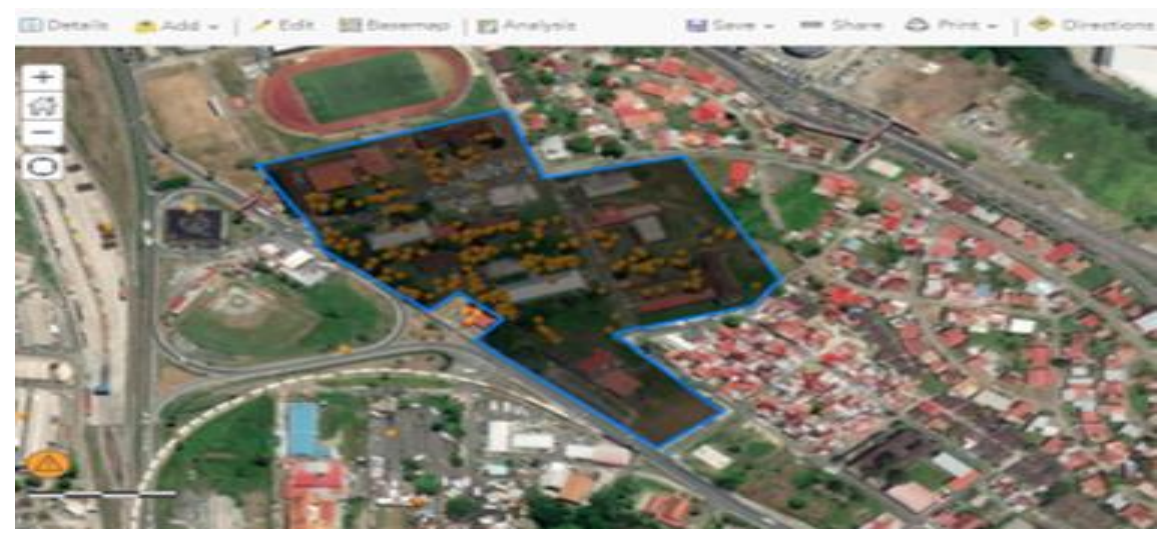

Figura 1. Medición del área del CRUC dado por Collector for Arcgis

El área de estudio se encuentra en una zona urbana cerca de bosques húmedos tropicales, característicos de la provincia de Colón. La temperatura promedio es de unos $28^{\circ} \mathrm{C}$ y varía durante el año. En esta región imperan dos temporadas: la seca y la de lluvias, siendo esta última, con precipitaciones abundantes. La humedad relativa promedio es de $80 \%$. Estas variaciones climáticas anuales provocan un panorama especial en el Centro Regional Universitario de Colón, existen áreas en las que se empoza el agua e igualmente se perciben cambios por razones de las características de cada especie (floración, frutos, caducifolios, entre otros). 


\begin{tabular}{|c|c|c|}
\cline { 2 - 3 } & REVISTA SABERES APUDEP & Volumen 3 Número 1 \\
\hline
\end{tabular}

Se inició con la observación de la distribución irregular de árboles de sombra y frutales, crecida sin control de herbazales, falta de atención del arbolado perimetral, sobre la base de la revisión del inventario florístico de árboles realizado entre 2012 y 2013 (Farnum y otros, 2014), para ubicar las especies de árboles en la extensión de las 14.9 hectáreas del Centro Regional Universitario de Colón.

Los datos del arbolado fueron obtenidos con un muestreo discrecional y capturando los datos en un formulario junto con su ubicación geográfica con el uso de la aplicación Collector for ArcGis, que es un "software" de Sistema de Información Geográfica diseñado por la empresa californiana Enviromental Systems Research Institute (ESRI) para trabajar a nivel multiusuario. Esta plataforma representa la evolución constante de estos productos, incorporando los avances tecnológicos experimentados en los últimos años en el área de la informática y telecomunicaciones para capturar, editar, analizar, diseñar, publicar en la web e imprimir información geográfica y relacionarla con otros aspectos en un espacio (Puerta, 2011), además ha demostrado un uso multifacético a la hora de la colecta de data científica con georreferenciación.

Evaluación fitosanitaria: Se realizó por observaciones sobre las características fisonómicas y características observables de los árboles inventariados, principalmente enfermedades ocasionadas de manera evidente por hongos, insectos $u$ otro tipo de infecciones, y daños mecánicos superficiales de origen antrópico como quiebres o cortes.

Consultas dialogadas: Se realizaron acercamientos con diferentes actores de la comunidad universitaria como profesores, grupos de estudiantes de diferentes carreras y asociaciones, con el fin de conocer sus diferentes puntos de vista sobre la utilidad y uso de las áreas verdes en el Centro Regional Universitario de Colón, siendo fuente importante de información. 


\begin{tabular}{|c|c|c|}
\cline { 2 - 3 } & REVISTA SABERES APUDEP & Volumen 3 Número 1 \\
\hline
\end{tabular}

\section{RESULTADOS Y DISCUSIÓN}

Durante el recorrido, se ubicaron árboles de sombra con árboles frutales sin una clara dinámica de interacción cerca de los edificios donde se imparten clases. En otros casos, se encontraron árboles muy altos cerca de edificios. También se ubicó una gran extensión arbórea en puntos donde no hay aulas de clases.

Con respecto al arbolado perimetral (Figura 2), se presume que tuvo como propósito absorber impactos contaminantes provenientes del tráfico de vehículos tales como ruidos, humo, smog, polvo y demás fuentes que atentan contra la salud humana y la salud ambiental. Durante la revisión de dicho arbolado, se observaron algunos con grandes deterioros lo que se presume, falta de atención. Por estas condiciones encontradas se recomienda hacer revisiones oportunas que aporten información útil al manejo y atención a las áreas verdes de nuestro centro universitario.

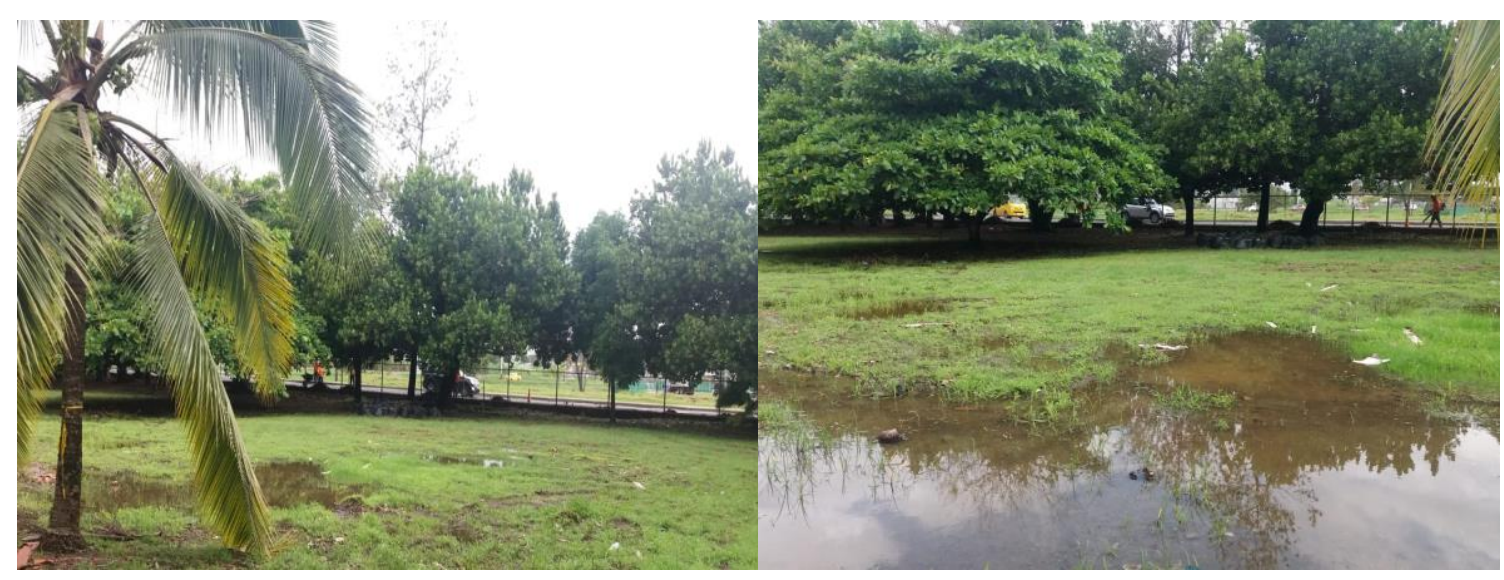

Figura 2, Árboles de la cerca perimetral. Centro Regional Universitario de Colón- Universidad de Panamá 


\begin{tabular}{|c|c|c|}
\cline { 2 - 3 } & REVISTA SABERES APUDEP & Volumen 3 Número 1 \\
\hline \\
\hline
\end{tabular}

Se encontraron zonas con crecida de herbazales, que supone ser la causa de estancamiento del drenado de aguas en la mayor parte del perímetro universitario, árboles con podas irregulares, áreas verdes subutilizadas e infraestructuras deterioradas por crecimiento de vegetación en zonas inapropiadas. (Figura 3a, 3b, 3c, $3 d)$
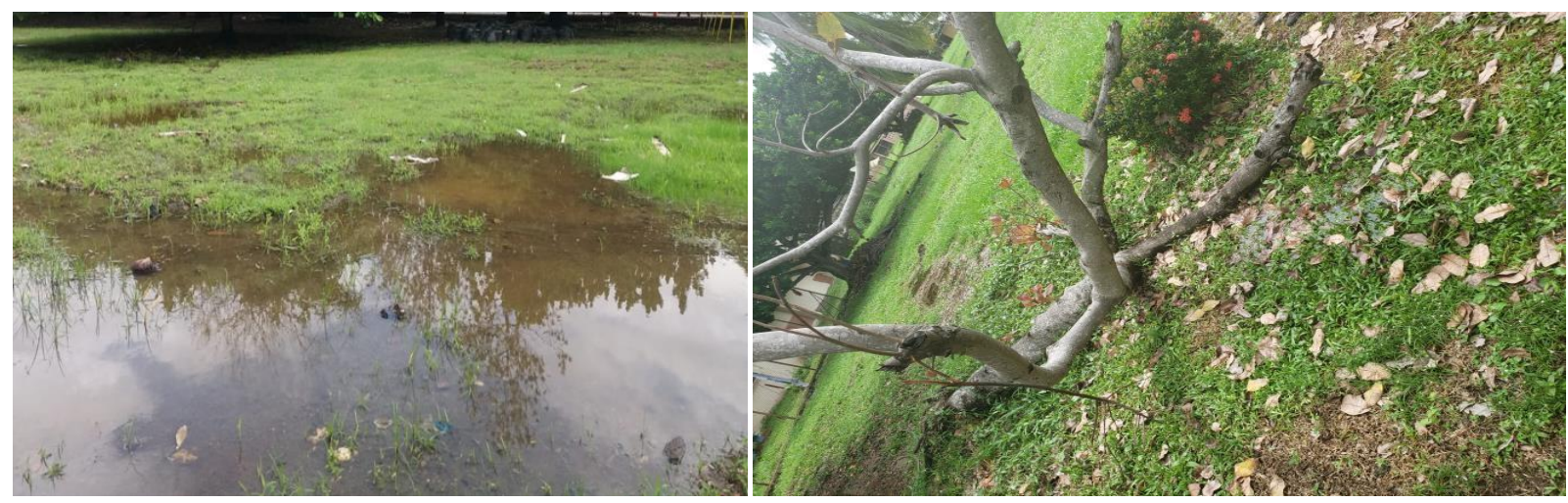

Figura 3a. Suelos inundados por problemas de drenaje, supone producto de la baja atención de las áreas verdes

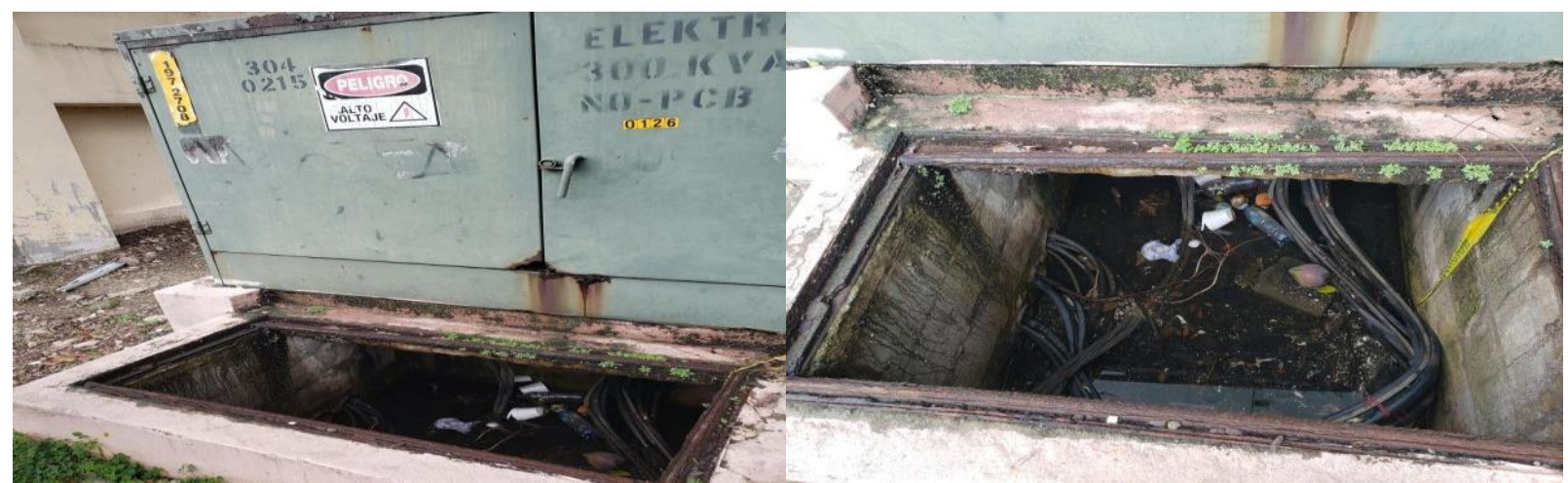

Figura 3b. Cajilla eléctrica sin seguridad dentro de las áreas verdes del Centro Regional Universitario de Colón. 


\begin{tabular}{|c|c|c|}
\cline { 2 - 3 } & REVISTA SABERES APUDEP & Volumen 3 Número 1 \\
\hline \\
\hline
\end{tabular}

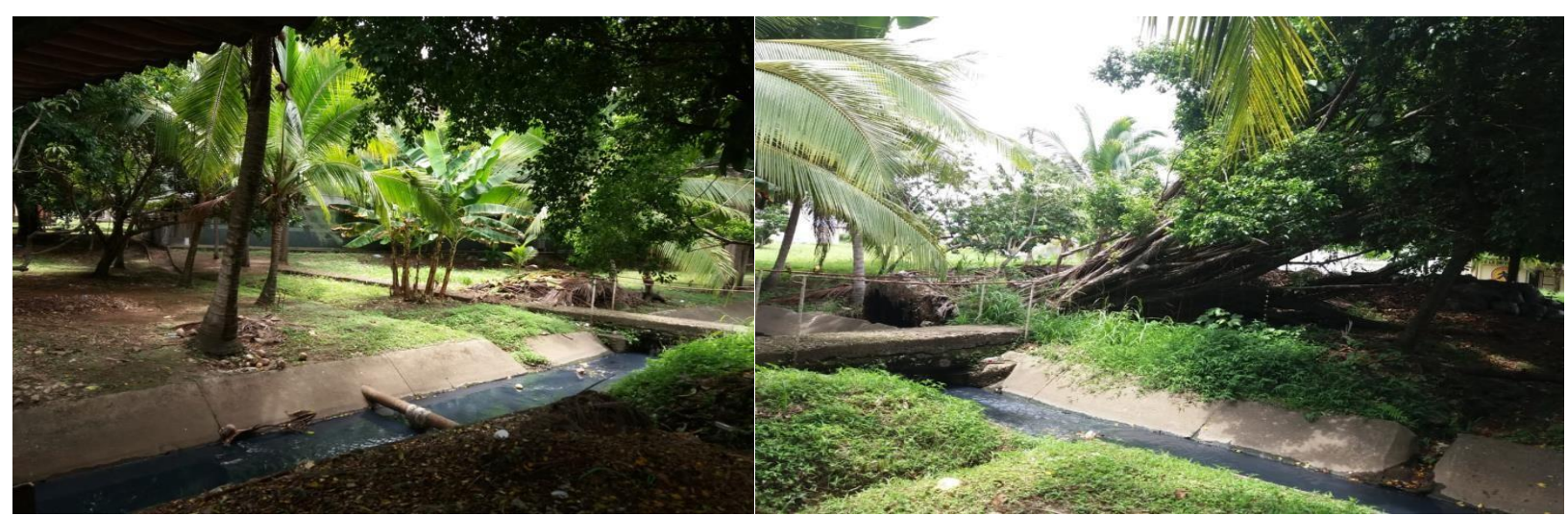

Figura 3c, cuneta de aguas residuales que atraviesa áreas verdes del Centro Regional universitario de Colón

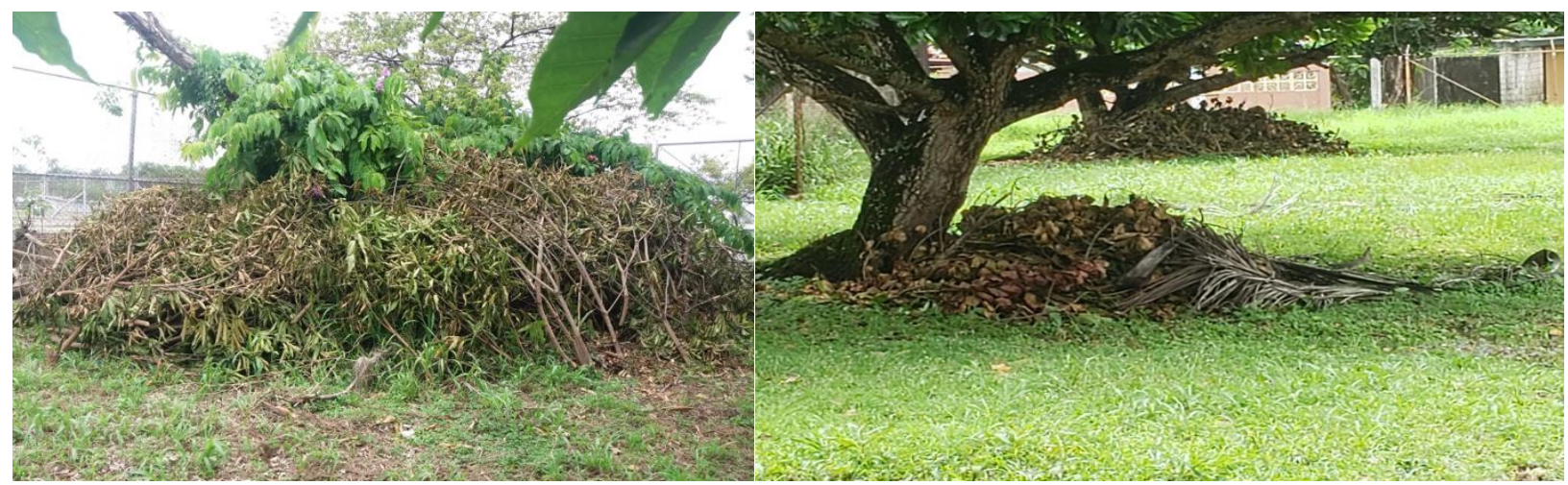

Figura 3d. Algunas áreas verdes subutilizadas del Centro Regional Universitario de Colón

Revisión del inventario florístico

A través de la revisión del inventario florístico (Figura 4), la observación de aspectos fitosanitarios y el análisis de abundancia y diversidad, se obtuvieron datos de composición, distribución y condiciones de las especies arbóreas dentro del Centro Regional Universitario de Colón. En este estudio, realizado de agosto a diciembre de 2019, se registró un total de 210 individuos, distribuidos en 27 especies arbóreas y 3 arbustivas.

El índice de Shannon para esta muestra fue de 1.3, mientras que el índice de diversidad de Simpson fue de 0,93584427, mostrando una dominancia baja de 0,06415573 . 


\begin{tabular}{|c|c|c|}
\cline { 2 - 3 } & REVISTA SABERES APUDEP & Volumen 3 Número 1 \\
\hline
\end{tabular}

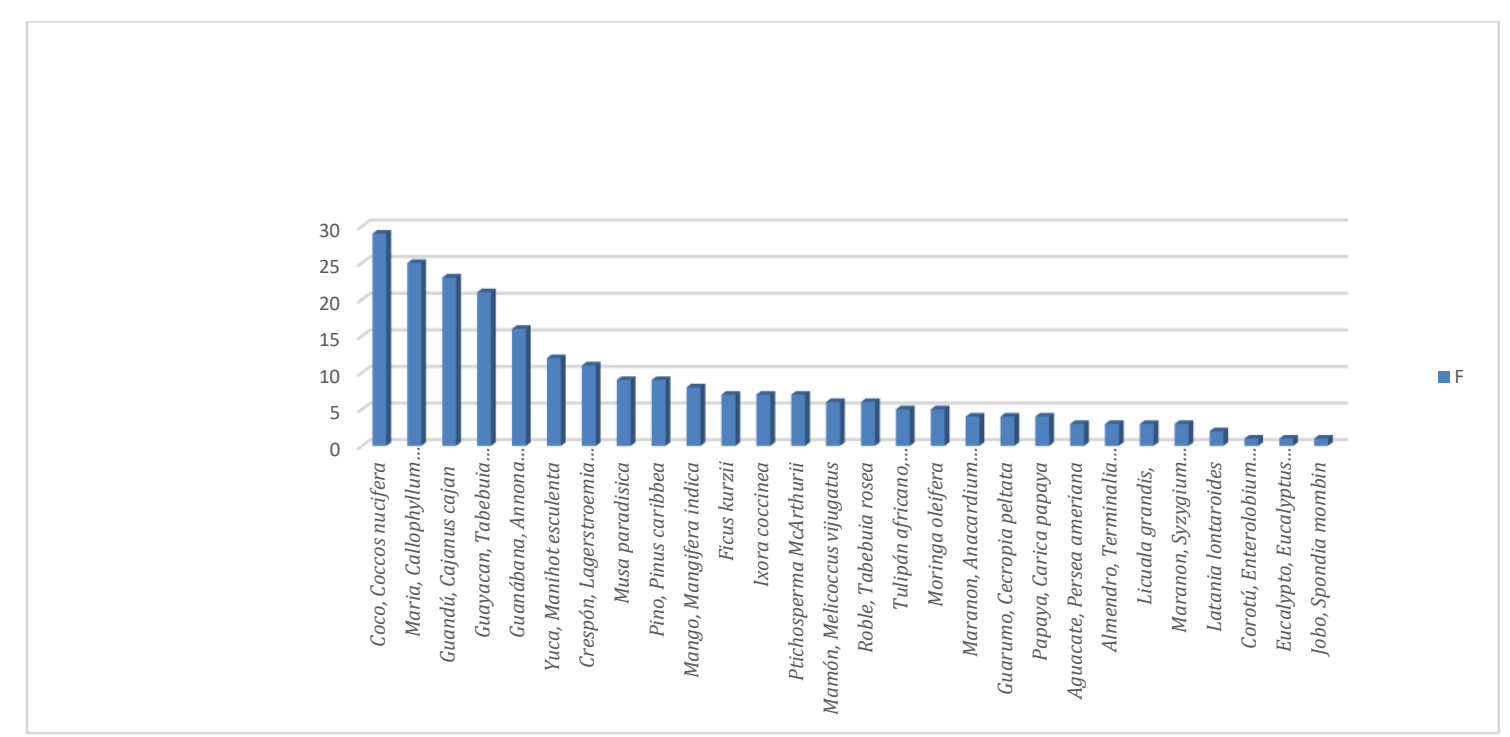

Figura 4. Especies de árboles en el Centro Regional Universitario de Colón

El resultado de nuestro inventario florístico, con referencia al 2014 (Farnum y otros), el cual había dado como resultado 60 especies, fue de 28 especies, lo que se traduce en una disminución del 53\%. La evidente diferencia de este resultado puede ser causada por las siguientes razones:

1) En estos momentos el centro se encuentra en una fase de construcción masiva y no se pudo acceder a ciertas áreas, lo que disminuyó el muestreo.

2) A diferencia de Farnum y otros, no tomamos en cuenta especies arbustivas (excepto Ixhora coccinea y Cajanus cajan) ni especies herbáceas

3) Nuestro tiempo de muestreo fue de 16 días a diferencia de Farnum y otros que tomaron alrededor de 1 año.

4) Algunas especies como Sterculia apetala y Swietenia macrophylla, han sido eliminadas 


\begin{tabular}{|c|c|c|}
\cline { 2 - 3 } & REVISTA SABERES APUDEP & Volumen 3 Número 1 \\
\hline
\end{tabular}

\section{Las especies}

Entre las especies más características o abundantes encontradas tenemos:

\section{Antrópicas}

1) Cocos nucífera: Una de las razones por la cual fue sembrada es porque que es una especie adaptable a ecosistemas tropicales.

2) Callophylum inophyllum: (Consulta Luciano Hernández, 2019) es uno de los árboles más abundantes en el Centro ya que fue sembrado en su perímetro.

3) Cajanus cajan: Fue implantado por su fruto (guandú) y por lo fácil de sembrar sus semillas.

4) Largestroemia: Fue sembrado como árbol ornamental en el lugar.

Distribuimos las especies muestreadas atendiendo a su uso cultural asociado a las especies más importantes (figura 5): artesanal (19.91\%), alimenticia (50.22\%), maderable $(26.84 \%)$, medicinal $(0.43 \%)$, ornamental $(2.6 \%)$.

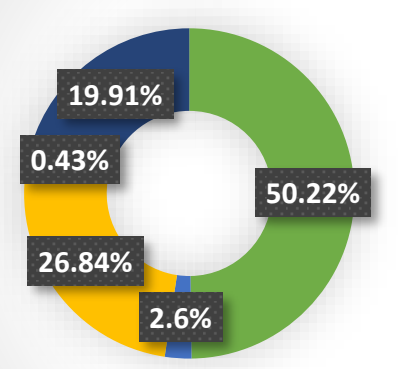

- Alimenticia

- Artesanal

Maderable

Medicinal

- Ornamental

Figura 5. Usos más comunes de los árboles del Centro Regional Universitario de Colón 
REVISTA SABERES APUDEP

ISSN L 2644-3805

Acceso Abierto. Disponible en:

https://revistas.up.ac.pa/index.php/saberes_apudep
Volumen 3 Número 1

Enero-Junio 2020

Recibido: $25 / 09 / 2019$

Aceptado: 13/12/2019

\section{Estado Fitosanitario}

En cuanto al estado fitosanitario, la afectación mayormente encontrada fue por causas antrópicas, incluyendo cortes, quiebres y contacto con artefactos. Igualmente se observaron un total de 56 árboles de la muestra con organismos patógenos, principalmente el comején (Termitidae). (Figura 6)
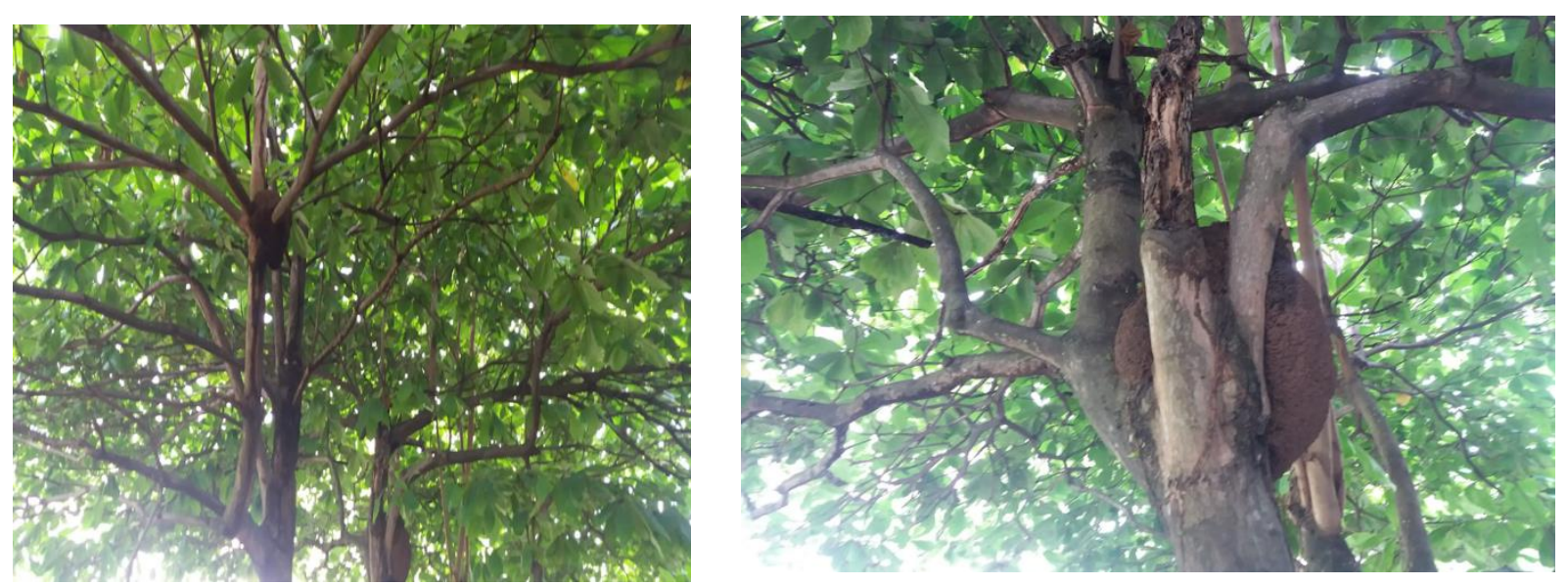

Figura 6. Presencia de comején en algunos árboles del Centro Regional Universitario de Colón.

El $72 \%$ de los árboles evaluados se encuentra en evidente mal estado fitosanitario (Figura 7); de estos, un 37\% muestra daños físicos antrópicos, posiblemente debido a una falta de mantenimiento adecuado que se refleja en cortes esporádicos, cosecha de frutos de manera descontrolada, no pocas veces con perjuicio de la planta.

Otro factor que llamó mucho la atención durante la colección de datos fitosanitarios fue la constante presencia de termitas en árboles de muchas especies diversas, las cuales son un signo de otros problemas a nivel de suelo, sobre todo aquellos relacionados con inundación (Boa, E. 2008). 


\begin{tabular}{|c|c|c|}
\cline { 2 - 3 } & REVISTA SABERES APUDEP & Volumen 3 Número 1 \\
\hline
\end{tabular}

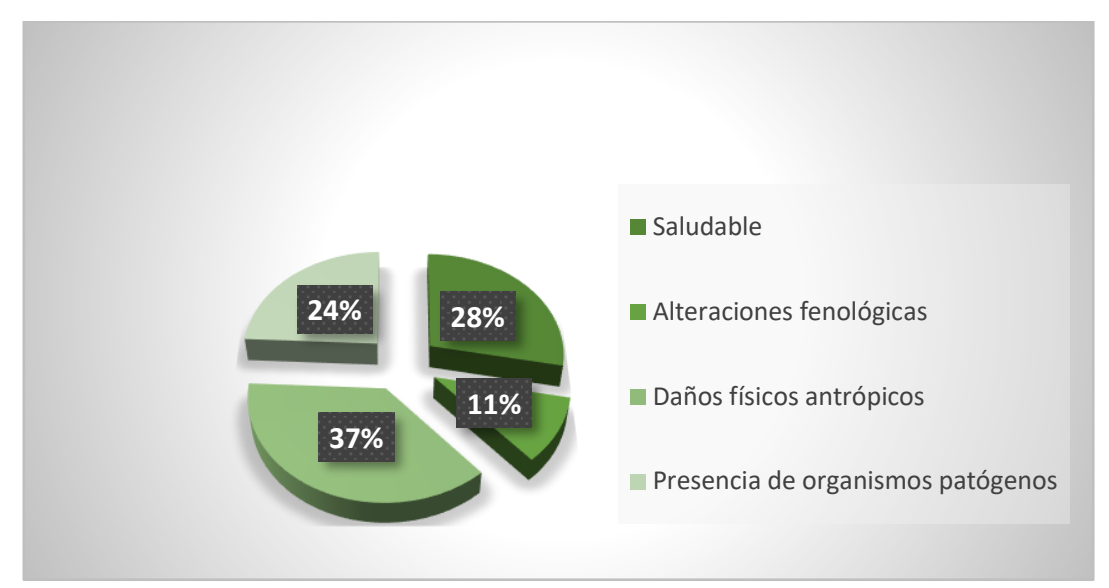

Figura 7. Estado fitosanitario de árboles Centro Regional Universitario de Colón

\section{Consultas dialogadas}

Las consultas realizadas con diferentes actores de la comunidad universitaria tales como profesores, administrativos y grupos de estudiantes de diferentes carreras y asociaciones, permitieron conocer diferentes puntos de vista sobre la utilidad y aprovechamiento de las áreas verdes en el Centro Regional Universitario de Colón. Se conocieron aspectos históricos del lugar que sirvieron para realzar su riqueza florística e ilustrar sobre las posibles causas que podían llevar a su deterioro. Dicha consulta arrojó información de acciones realizadas en otrora con el propósito de rescatar estos espacios en beneficio del centro universitario.

Entre estas acciones según el biólogo Luciano Hernández, está el hecho de que, para la época de los años `90 se realizó la siembra de la cerca perimetral, lo que supone era para amortiguar algunos impactos ambientales. El Mgter. Carlos Becerra manifestó que años más tarde, junto con el Profesor Pedro Mc Claod se realizó la siembra de algunos árboles frutales en el área de la Facultad de Humanidades, lo que supone una actividad de la escuela de geografía para acondicionar un sendero dentro del centro universitario, como área de trabajo de campo. 


\begin{tabular}{|c|c|c|}
\cline { 2 - 3 } & REVISTA SABERES APUDEP & Volumen 3 Número 1 \\
\hline
\end{tabular}

Por su parte, los administrativos del departamento de mantenimiento de áreas verdes del Centro Regional Universitario de Colón, señalan que atienden las instrucciones de corte de hierba y poda cuando se les indica. Debido a toda la logística (equipos, combustibles, disposición final de desechos productos de la actividad) que esto genera, se supone una falta de programación para este propósito.

\section{CONCLUSIÓN}

Partiendo de la revisión del ya existente inventario arbóreo del Centro Regional universitario de Colón realizado en el año 2014 por el Dr. Francisco Farnum y otros, el presente estudio ha permitido constatar que algunas especies han desaparecido de este lugar.

Hemos observado que más del $70 \%$ de los organismos vegetales arbóreos del centro universitario se encuentran en mal estado de salud, sobre todo por intervención antrópica, lo que conduce a presumir la falta de un plan de manejo para dichas especies. (Figura 6, Figura 7).

Cabe señalar que en las instalaciones del Centro Regional Universitario de Colón se iniciaron con mejoras y remozamiento, a partir del año 2017. Es imperante implementar un plan de manejo de áreas verdes, que permita conservar las especies vegetales que se encuentran en nuestra universidad y que brindan servicios ambientales, no solamente en el rol dentro del ecosistema sino como una característica esencial para la buena calidad de vida y como parte importante de atención en las necesidades de un centro de formación profesional.

Por tal razón, se recomienda aprovechar la información que muestra este estudio para poner en ejecución una gestión ambiental que conduzca a la implementación de estrategias de sensibilización a la comunidad universitaria y prevenga la degradación total de estas áreas. 


\begin{tabular}{|c|c|c|}
\cline { 2 - 3 } & REVISTA SABERES APUDEP & Volumen 3 Número 1 \\
\hline
\end{tabular}

Para ello es necesario que toda la comunidad universitaria del Centro Regional Universitario de Colón, se empodere de la situación, de modo tal, que ofrezcan sostenibilidad a este recurso natural con que se cuenta.

\section{REFERENCIAS BIBLIOGRÁFICAS}

Boa, Eric. Guía llustrada sobre el estado de salud de los árboles. Reconocimiento e interpretación de síntomas y daños. El Salvador, 2008- pp 4-6

Caballero Deloya, M. 1996. Urban Forestry Activities in México. Journal Arboriculture. 12 (10): 251-256.

Correa, M.; Stapf, M.; De Sedas, A.; Hernández, F. y Carranza, R. 2010. Árboles y Arbustos del Parque Natural Metropolitano de Panamá.

De Sedas, A.; Hernández, F.; Carranza, R.; Stapf, M. y Correa, M. 2010. Guía de Árboles y Arbustos del Campus Dr. Octavio Méndez Pereira, Universidad de Panamá.

Escudero, J. y Gross, P. 1996. Deterioro ambiental en los asentamientos humanos, "Chile Urbano". Antecedentes de la consulta Nacional para la formulación de desarrollo urbano 1993-1996. Ministerio de Vivienda y Urbanismo. Santiago, Chile.

Farnum, F.; Ayala, J.D.; Sánchez, G. y Murillo, V. 2014. Estudio de la diversidad de árboles y arbustos en los predios del Centro Regional Universitario de Colón. Revista Colón Ciencias. 1(1):43-56.

Grey, G.W. y Deneke, F.J. 1992. Urban Forestry. Krieger Publishing Company. United States Department of Agriculture. Malabar, Florida, U.S.A. 299p.

Ríos Cáceres, S.; Pogois Loayza, D.; Ríos Bermúdez, F.; Carrera La Torre, B. y Díaz Ching, M. 1998. Estrategias aplicables a la Gestión Ambiental de Áreas Verdes Urbanas. Instituto Nacional de Protección del Medio Ambiente para la salud. Lima, Perú. 102.

Rodríguez Salgado, M.C. 2012. Manejo de Áreas Verdes en Concepción: Mejor Calidad de Vida Urbana. Calidad de Vida Urbana Sectorial / Urbano. 41-47 


\begin{tabular}{|c|c|c|}
\cline { 2 - 3 } & REVISTA SABERES APUDEP & Volumen 3 Número 1 \\
\hline
\end{tabular}

Undurraga, C. 1984. Ornato y Urbanismo, guía para alcalde y otros funcionarios. Editorial Morgan Marinetti. Santiago, Chile.

Universidad de Panamá 2019. -Dirección de Tecnología de la información y Comunicación. www.crucolon.up.ac.pa

XI Congreso Mundial Forestal. 1997. Silvicultura Urbana y Periurbana. Volumen 1. Tema 3. Antalya, Turquía. 